\title{
APPLICATION OF EXAFS TO CATALYSIS AND SURFACE SCIENCE
}

\author{
A.J. RENOUPREZ \\ Institut de Recherches sur la Catalyse, 2 av. A. Einstein, 69626 Villeurbanne, France
}

The paper gives recent applications of EXAFS to the structural study of microcrystalline bimetaltic particles. Special attention has been paid to the case of systems composed of neighbouring elements Also, it is shown how the use of X-ray diffraction techniques and surface science methods like ion scattering can be indispensable to interpret the EXAFS data correctly. In catalysis and surface science, experiments performed in total reflection mode can provide a detailed description of the adsorption site of atoms on a flat surface.

PACS numbers: $61.10 . \mathrm{Lx}, 78.70 . \mathrm{Dm}$

\section{Introduction}

Metallic catalysts are currently composed of two metals deposited on the surface of an inorganic oxide or inserted in the network of this oxide. The degree of interaction between these two elements and the possible formation of alloys are uneasily studied by diffraction techniques because of the low concentration of the metal and of the small diameter of the particles. Due to its spectroscopic character and its high sensitivity when performed with synchrotron radiation, EXAFS has proved to be of great help in solving this problem. The case of the association of two neighbouring atoms in periodic classification is however one of the most difficult but now can be tackled. In some cases where X-ray diffraction is possible, because of a large difference in atomic radius of the two elements, a comparison can be instructive to check the limitation of the two methods. Also the composition of the outmost surface can in principle be determined by EXAFS provided that the measurements are precise enough but a comparison with the actual surface composition measured by low energy ion scattering shows the limitations of EXAFS. Another example of interesting new type of study is the structural investigation of small metallic clusters deposited on oriented substrates, using the polarization of the radiation in total reflection mode, to determine the adsorption site of the cluster on the surface. 


\section{Structural study of bimetallic clusters}

During the last years many Pt or Pd based bimetallic systems have been studied by EXAFS [1-5]. Among various studies, the work of a Japanese group with Toshima, Harada et al. has been one of the most successful $[1,2]$. They have studied colloidal dispersions of $\mathrm{Pd}-\mathrm{Pt}$ and $\mathrm{Pd}-\mathrm{Rh}$ clusters which are catalysts for partial hydrogenation of unsaturated hydrocarbons. The metallic particles are protected from aggregation by a coating with a polymer, added during the preparation. Two different $\mathrm{Pd}-\mathrm{Pt}$ samples [1] were studied with a $1 / 1$ and $4 / 1$ atomic ratio of the elements. As shown in Fig. 1 , the $\mathrm{Pd}_{50} \mathrm{Pt}_{50}$ clusters have the same activity as pure palladium in the hydrogenation of cyclooctadiene to cyclooctene but the $\mathrm{Pd}_{80} \mathrm{Pt}_{20}$ clusters are three times more active. In both cases, the mean diameter measured by electron microscopy is approximately $1.5 \mathrm{~nm}$.

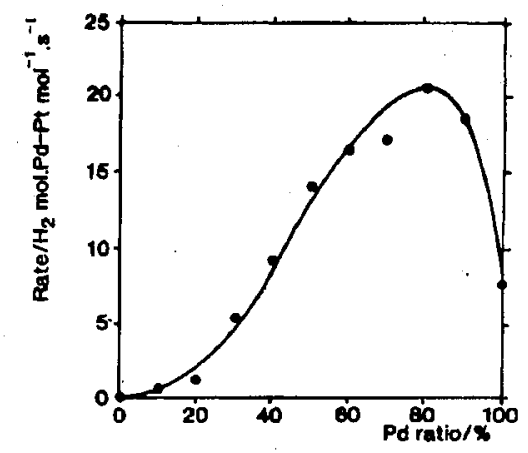

Fig. 1. Rate of hydrogenation of the cyclooctadiene as a function of the composition of the Pd-Pt clusters.

The EXAFS study of these clusters at both edges has been performed on concentrated suspensions. An important point is the use of $10 \mu \mathrm{m} \operatorname{Pt}_{90} \mathrm{Pd}_{10}$, $\mathrm{Pd}_{90} \mathrm{Pt}_{10}$ alloy foils and pure metal foils as reference compounds to extract $\sigma$, the Debye-Waller factor, $\Delta E$, the edge shift with respect to its theoretical position, and the amplitude reduction factor $S$. In the fitting procedure of the monometallic reference compounds and of the unknown clusters, they have used the theoretical phase shifts and back-scattering amplitudes calculated by Teo and Lee [6]. It can be seen in Table I that the values found for $N$ and $R$, the coordination number $(C N)$ and the interatomic distance, correspond to those obtained from crystallography. It also appears that on clusters, they found $\Delta E$ values similar to those of the foils but $\sigma$ was slightly larger. In the final analysis of the bimetallic clusters they have kept for $\Delta E, S$ and $\sigma$ the values found for the pure metal clusters.

In Table II, the results of the fit on the $4 / 1 \mathrm{Pd}-\mathrm{Pt}$ cluster are compared by the authors to two possible models of 55 atoms particles. In the case where the two kinds of atoms are randomly distributed in the particle, Fig. $2 \mathrm{~b}$, one expects a number of neighbouring $\mathrm{Pt}$ atoms around each element to be three times less than the number of $\mathrm{Pd}$ atoms, which is not confirmed by the experiment. A 
TABLE I

EXAFS analyses for the model reference compounds.

\begin{tabular}{l|l|l|c|c|c|c}
\hline Reference & Edge & Bond & $N$ & $r[\AA]$ & $\Delta E[\mathrm{eV}]$ & $\sigma[\AA]$ \\
\hline Pd foil & Pd $K$ & Pd-Pd & 12 & 2.73 & -10.3 & 0.066 \\
Pt foil & Pt $L$ & Pt-Pt & 12 & 2.76 & -2 & 0.059 \\
Pd cluster & Pd $K$ & Pd-Pd & 6.2 & 2.76 & -12.7 & 0.074 \\
Pt cluster & Pt $L$ & Pt-Pt & 8 & 2.75 & 0.8 & 0.065 \\
Pd/Pt 1/9 & Pd $K$ & Pd-Pt & 12 & 2.76 & -9.3 & 0.066 \\
foil & & & & & & \\
Pd/Pt 9/1 & Pt $L$ & Pt-Pd & 12 & 2.73 & 0.9 & 0.062
\end{tabular}

TABLE II

EXAFS results and comparison with models for the bimetallic cluster with $4 / 1$ composition.

\begin{tabular}{c|c|c|c|c|c}
\hline \hline \multicolumn{2}{c|}{} & \multicolumn{3}{c}{ Coordination number } \\
\hline Absorber & Scatterer & $\begin{array}{c}\text { Distance } \\
{[\AA]}\end{array}$ & Measured & $\begin{array}{c}\text { Pt core } \\
\text { model }\end{array}$ & $\begin{array}{c}\text { Random } \\
\text { model }\end{array}$ \\
\hline $\mathrm{Pd}$ & $\mathrm{Pd}$ & 2.74 & 4.4 & 4.6 & 6 \\
$\mathrm{Pd}$ & $\mathrm{Pt}$ & 2.73 & 2.3 & 2 & 1.9 \\
$\mathrm{Pt}$ & $\mathrm{Pt}$ & 2.73 & 5.5 & 5.5 & 1.9 \\
$\mathrm{Pt}$ & $\mathrm{Pd}$ & 2.72 & 3.5 & 3.5 & 6
\end{tabular}

second model, Fig. 2a, is alternatively considered, composed of a core of $13 \mathrm{Pt}$ atorns surrounded by $42 \mathrm{Pd}$ atoms. In that case, the $C N$ around $\mathrm{Pt}$ is close to 12 and 7 around $\mathrm{Pd}$. The experimental values, 9 and 6.7, militates in favour of this second model, taking into account the error bars on these numbers and the possible presence of a small number of $\mathrm{Pt}$ atoms on the surface. Moreover, it is known from theoretical predictions that in this alloy $\mathrm{Pd}$ has a strong tendency to migrate at the surface. For the cluster with a $1 / 1$ atomic composition, the results shown in Table III indicate, as mandated by the composition, a decrease in the
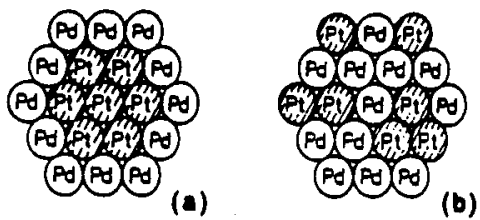

Fig. 2. Clusters with a 4/1 atomic composition; (a) Pt core model and (b) random model. 
TABLE III

Comparison of EXAFS results with three possible models for the bimetallic clusters $\mathrm{Pd}-\mathrm{Pt}$ with the $1 / 1$ composition.

\begin{tabular}{c|c|c|c|c|c|c}
\hline \hline \multicolumn{2}{|c|}{} & \multicolumn{4}{c}{ Coordination numbers } \\
\hline Absorber & Scatterer & Distance $[\AA]$ & Measured & Pt core & Random & Dimixion \\
\hline $\mathrm{Pd}$ & $\mathrm{Pd}$ & 2.4 & 2.3 & 3.1 & 3.9 & 6 \\
$\mathrm{Pd}$ & $\mathrm{Pt}$ & 2.74 & 3 & 3 & 3.9 & 1.7 \\
$\mathrm{Pt}$ & $\mathrm{Pt}$ & 2.73 & 4.8 & 6.6 & 3.9 & 6.4 \\
$\mathrm{Pt}$ & $\mathrm{Pd}$ & 2.73 & 1.3 & 2.9 & 3.9 & 1.6
\end{tabular}

number of $\mathrm{Pd}$ atoms around both $\mathrm{Pt}$ and $\mathrm{Pd}$ atoms. In that case the authors conclude that all the $\mathrm{Pt}$ atoms remain interconnected, forming a 28 atoms core and that the $27 \mathrm{Pd}$ atoms forms islands at the surface of this particle (Fig. 3a). This accounts for the reduction of 9 to 6.1 of the $C N$ around $\mathrm{Pt}$ compared to the previous case and for the low value of 5.3 around $\mathrm{Pd}$. The model where the atoms would be randomly distributed in the particle, shown in Fig. $3 \mathrm{~b}$, is rejected because it leads to a constant value of the $C N$ around each type of atom. Finally, the model represented in Fig. 3c, with a total separation of the two phases is physically less probable and would lead to larger $\mathrm{Pd}-\mathrm{Pd} C N$.

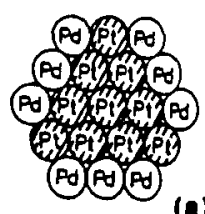

(a)

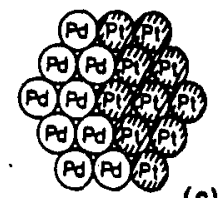

(c)

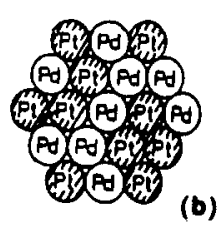

(b)

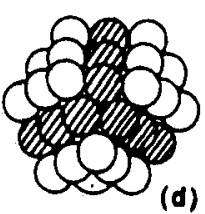

Fig. 3. Clusters with a $1 / 1$ atomic composition; (a) the modified Pt core model, (b) the random model, (c) the model with dimixion, and (d) a three-dimensional representation of model (a).

The difference of reactivity observed between these two clusters $[7]$ is, for these authors, well explained by the structural differences. Indeed they assume that the most reactive species are constituted by $\mathrm{Pd}$ atoms electronically modified by underlying $\mathrm{Pt}$ atoms, a configuration found in the $4 / 1$ cluster. On the contrary, the $\mathrm{Pt}$ atoms directly located on the surface and mainly in interaction with $\mathrm{Pt}$ atoms, would be less reactive. This corresponds to the case of the $1 / 1$ cluster.

The same group [2] was facing a still more complex problem in the EXAFS 
study of palladium-rhodium clusters. Indeed, the main difficulty arises from the fact that for two neighbouring elements, the backscattering amplitudes are very similar and the distinction between the two different elements in the same layer is hazardous. Moreover, microprobe analysis indicates that the situation is complex: besides the $2.5 \mathrm{~nm}$ bimetallic clusters, the samples contain pure Rh clusters of less than $2 \mathrm{~nm}$ and larger Pd particles of $3.5 \mathrm{~nm}$. This is a typical situation where EXAFS alone is not able to lead to a complete description of the local order in the bimetallic particles.

In a recent study of silica supported Pd-Ni bimetallic particles, Faudon et al. [4] have shown that a complete description of such systems can be obtained by using simultaneously crystallography and X-ray absorption methods. The clusters deposited on a silica support were obtained in two steps. Monometallic Pd particles were first prepared by the decomposition followed by a reduction under hydrogen of an aminocomplex, $\mathrm{Pd}\left(\mathrm{NH}_{3}\right)_{4}(\mathrm{OH})_{2}$, deposited on the silica. A nickel complex, $\left(\mathrm{C}_{5} \mathrm{H}_{5}\right)_{2} \mathrm{Ni}$, was then grafted on the palladium particles by exchange of the $\mathrm{C}_{5} \mathrm{H}_{5}$ ligands with hydrogen atoms chemisorbed on the palladium. After a thermal treatment under hydrogen, one obtains a bimetallic phase. Under such conditions, EDX STEM shows that no pure nickel particles are found on the silica and that all the bimetallic particles have the same composition within $2 \%$. The particles observed by electron microscopy have a diameter of 5 to $7 \mathrm{~nm}$ and thus can be studied by X-ray diffraction in spite of the fact that the metal concentration is not larger than $3 \mathrm{wt} . \%$.

The X-ray spectra were recorded with a linear position sensitive detector covering an angular range of $12^{\circ}$. It was however necessary to scan a large range of $30^{\circ}$ to subtract the background of the silica support, which constitutes $90 \%$ of the signal. The sample is placed in an oven which can be heated up to $900 \mathrm{~K}$ under controlled atmosphere. The diffractograms obtained under hydrogen at 490 and $720 \mathrm{~K}$ are represented in Fig. 4 for three different compositions of the precursors. For the 15 and $77 \mathrm{Pd}$ atomic concentrations, the alloy is already formed at $490 \mathrm{~K}$ and becomes homogeneous after a treatment at $720 \mathrm{~K}$. This is confirmed by the width of the diffraction lines corresponding to crystal diameters of $7 \mathrm{~nm}$, in agreement with electron microscopy measurements. For the $\mathrm{Pd}_{40} \mathrm{Ni}_{60}$ composition, two phases are clearly visible at $490 \mathrm{~K}$ and a distribution of composition persists at $720 \mathrm{~K}$, since the line width gives a crystallite size of $4 \mathrm{~nm}$ and the electron microscopy, $6 \mathrm{~nm}$.

The results of the EXAFS experiments performed at both edges are gathered in Tables IV and V. A first observation is the consistency between the observations at both edges. The Ni-Pd and $\mathrm{Pd}-\mathrm{Ni}$ distances measured at the $\mathrm{Ni}$ and $\mathrm{Pd}$ edges, respectively, are equal within an accuracy of $1 \%$. Also the total coordination numbers vary from 10.5 to 10.8 , which is consistent with the diameter of particles composed of 1500 to 2000 atoms. It is also known [8] that the coordination numbers $N$, and the concentration $C$, for the atoms $\mathrm{A}$ and $\mathrm{B}$ are related by the expression

$$
C_{\mathrm{A}} N_{\mathrm{AB}}=C_{\mathrm{B}} N_{\mathrm{BA}}
$$

leading to $\mathrm{Pd}$ concentrations of $0.13,0.53$ and 0.80 compared to $0.15,0.40$ and 0.77 as determined by the chemical analysis. A final point is that the total $C N$ 

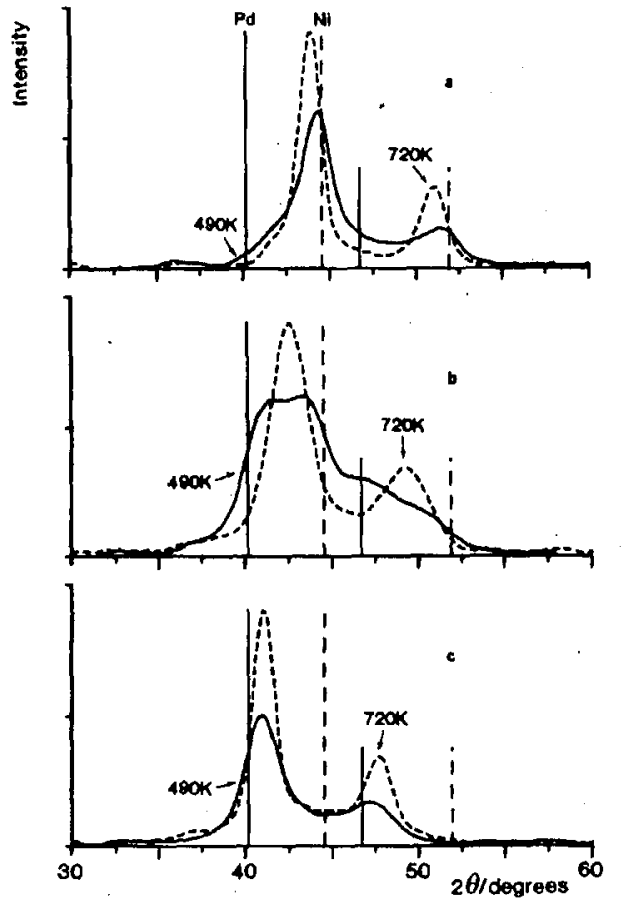

Fig. 4. X-ray patterns of $\mathrm{Pd}-\mathrm{Ni}$ samples reduced at 490 and $720 \mathrm{~K}$. (a), (b) and (c) for atomic $\mathrm{Pd}$ concentrations of 15,40 and $77 \%$ respectively.

TABLE IV

EXAFS results on $\mathrm{Pd}-\mathrm{Ni}$ clusters at the $\mathrm{Pd} K$-edge.

\begin{tabular}{c|c|c|c|c|c}
\hline \hline Sample & \multicolumn{2}{|c|}{ Pd-Pd pairs } & \multicolumn{2}{|c|}{ Pd-Ni pairs } & \\
\cline { 2 - 6 } & $N_{1}$ & $R_{1}$ & $N_{2}$ & $R_{2}$ & $N_{1}+N_{2}$ \\
\hline Pd foil & 12 & 2.76 & & & \\
Pd cluster & 6.3 & 2.76 & & & \\
Pd-Ni 15/85 & 1.5 & 2.70 & 9.3 & 2.49 & 10.8 \\
Pd-Ni 40/60 & 5 & 2.70 & 5.5 & 2.48 & 10.5 \\
Pd-Ni 77/23 & 8.3 & 2.70 & 2.4 & 2.44 & 10.7
\end{tabular}

around the two elements is similar, excluding a segregation of one of the elements for these samples equilibrated below $800 \mathrm{~K}$. But one has however to consider the problem of accuracy of the determination of the $C N$ and the fact that only the first coordination shell is considered. Indeed if a segregation occurs (Pd in this case), the surface atoms expected to have a reduced number of first neighbours, only constitute 15 to $18 \%$ of the total number of atoms of the particle. So a precision better than $5 \%$ in the determination of $N$ would be necessary to confirm a possible segregation. It is known that this accuracy is rather $10 \%$. 


\section{TABLE V}

EXAFS results on the $\mathrm{Pd}-\mathrm{Ni}$ clusters at the $\mathrm{Ni}$ $K$-edge.

\begin{tabular}{c|c|c|c|c|c}
\hline \hline \multirow{2}{*}{ Sample } & \multicolumn{2}{|c|}{ Ni-Pd pairs } & \multicolumn{2}{|c|}{ Ni-Ni pairs } & \\
\cline { 2 - 6 } & $N_{1}$ & $R_{1}$ & $N_{2}$ & $R_{2}$ & $N_{1}+N_{2}$ \\
\hline Ni foil & & & 12 & 2.49 & \\
Pd-Ni 15/85 & 1.3 & 2.57 & 9.3 & 2.49 & 10.6 \\
Pd-Ni 40/60 & 6.4 & 2.61 & 4.4 & 2.48 & 10.8 \\
Pd-Ni 77/23 & 9.5 & 2.64 & 1.3 & 2.44 & 10.8
\end{tabular}

To obtain a precise evaluation of the extent of the superficial segregation, low energy ion scattering (LEIS) experiments were performed on these samples. For this purpose $1 \mathrm{keV}^{4} \mathrm{He}$ ions are sent on the sample where they undergo inelastic collisions. The ratio of the energies of the impinging and scattered ions, $E_{1} / E_{0}$, leads to $\gamma$ - the ratio of the mass of the surface atoms and incident ions

$$
E_{1} / E_{0}=\left\{\left[\cos \theta+\left(\gamma^{2}-\sin ^{2} \theta\right)^{1 / 2}\right] /(1+\gamma)\right\}^{2},
$$

where $\theta$ is the scattering angle. This relation leads to the identification of the surface atoms. The intensity of the peaks located at a well-defined energy (775 and $865 \mathrm{eV}$ for $\mathrm{Ni}$ and $\mathrm{Pd}$, in the present case), are calibrated by comparison with single crystal faces of the pure elements with known orientations. This leads to the composition of the first layer.

Actually these low energy ions only probe the first layer because they are very rapidly neutralized after the first collision with surface atoms [9]. However they are able to remove the atoms of this first layer and this sputtering should normally modify the measured surface composition for long times of irradiation. Indeed this phenomenon is observed on Fig. 5 corresponding to a sample containing only $9 \mathrm{Pd}$ at.\%. The surface concentration measured by LEIS is $47 \%$, but decreases to $20 \%$ after 40 min of sputtering. It is also evidenced that the temperature of equilibration has an influence on this surface concentration. Actually, the difference observed between 820 and $920 \mathrm{~K}$ is purely due to kinetic effects, since the thermodynamic would predict the reverse temperature effect.

This segregation of palladium can be predicted on simple thermodynamic grounds, in an approach described by Winblatt and Ku [10] for example. This method minimizes the free energy of the system taking into account (i) the surface tensions depending on the sublimation heats, (ii) the elastic strain favouring the migration of the biggest atom and (iii), the free enthalpy of mixing. Here the first two factors favour the migration of Pd at the surface and the last term, which is small, has a limited influence.

In small particles however the reservoir of diffusing atoms is limited by its mean concentration. The consequence is that if the particles have a diameter of less than $1.5 \mathrm{~nm}$ for example, and have thus $50 \%$ of the atoms on the surface, the surface concentration of the segregating species will remain below $50 \%$ for the alloys which do not reach the nominal 50/50 atomic composition. This is expressed 


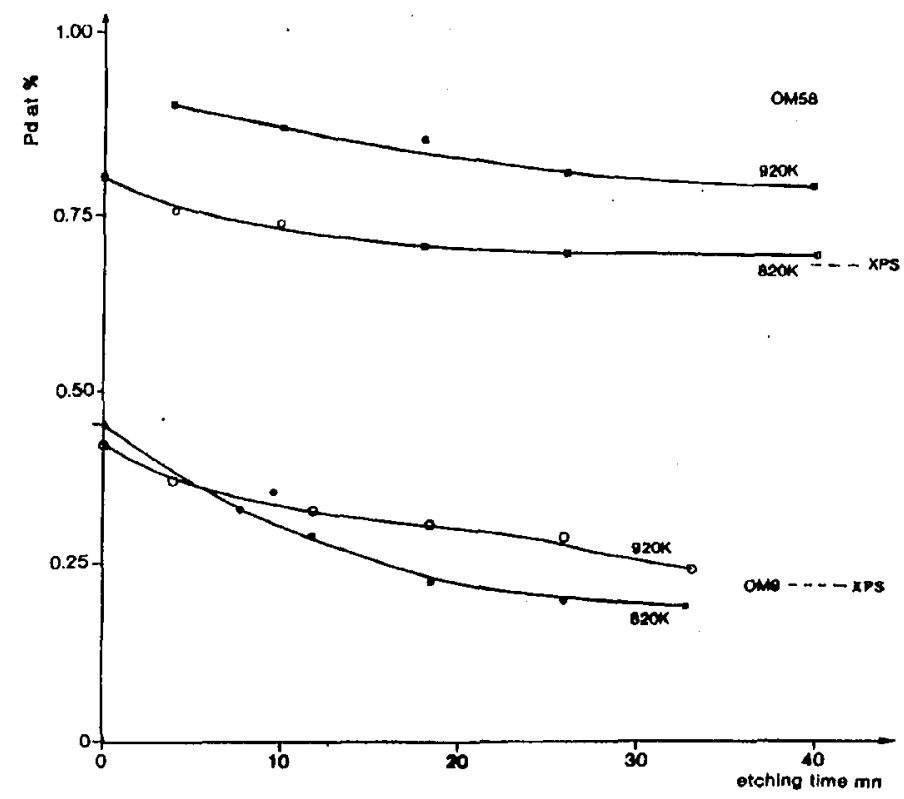

Fig. 5. Surface composition measured by LEIS as a function of the sputtering time, for samples with nominal compositions of 9 and $58 \mathrm{Pd}$ at.\%.

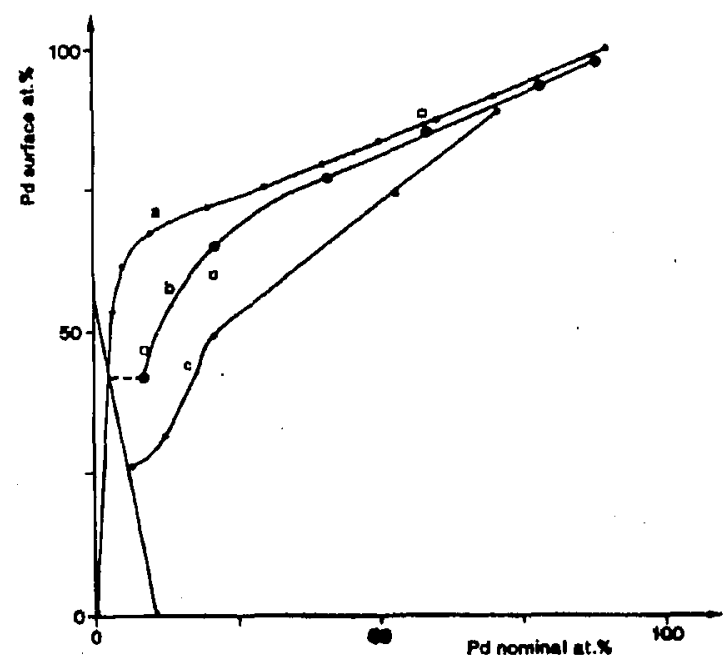

Fig. 6. Surface composition predicted by thermodynamic calculations. (a) for bulk alloys, (b) for particles with $15-20 \%$ and (c) with $40-50 \%$ dispersion.

by the simple relation

$$
X_{\mathrm{N}}=D X_{\mathrm{S}}+(1-D) X_{\mathrm{B}}
$$


where $X_{\mathrm{N}}, X_{\mathrm{S}}$ and $X_{\mathrm{B}}$ are the nominal, surface and bulk concentrations and $D$ - the ratio between the number of surface and the total number of atoms.

In Figs. $6 \mathrm{a}$ and $6 \mathrm{~b}$ this surface concentration is represented for bulk alloys and particles with values of $D$ ranging from 15 to $20 \%$. The square symbols, corresponding to LEIS measurements, agree remarkably with the predictions. It appears that as expected, the corrections for the dispersion of the particles, which lowers the actual surface concentration, has only an effect for the low nominal concentrations. Also it can be observed in Fig. 6c corresponding to particles of 35 to $40 \%$ dispersion (not described here) that the $\mathrm{Pd}$ surface concentration is strongly decreased for these smaller particles, especially at low nominal concentrations.

Hence it is clear that the EXAFS even if it remains a powerful method is not able alone to provide a complete description of the structure of these bimetallic particles. Indeed, the determination of the coordination numbers is not precise enough to confirm a segregation of one of the elements at the surface.

\section{Structural studies by EXAFS in reflection mode}

Since the early work of Citrin et al. [11], it has been known that flat surfaces including several elements can be structurally studied in reflection mode, using the fact that synchrotron radiation is linearly polarized. More precisely, the site of adsorption of an atom $\mathrm{A}$ on a surface composed of atoms $\mathrm{B}$ can be determined by performing two EXAFS experiments at the edge of A with the electric-field vector $\varepsilon$ of the incident beam parallel and perpendicular to the surface.

Actually the effective coordination number given by each experiment would be

$$
N_{i}=3 \sum \cos ^{2} \alpha_{i},
$$

where $\alpha$ is the angle between the electric-field vector $\varepsilon$ and the bond vector $r_{i}$. In the example of Fig. 7 of an adsorbate located on a surface with a (111) orientation,

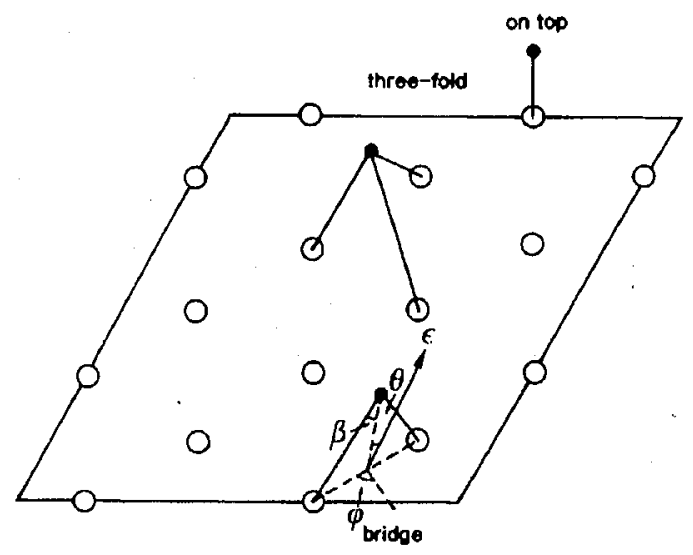

Fig. 7. Adsorbate on three types of sites on a (111) face. For the represented bridge sites, $\theta$ is the angle between the electric-field vector $\varepsilon$ and the surface normal. 
the atoms located on top of substrate atoms would not contribute to the EXAFS signal for electric-field vectors parallel to the surface ( $p$ polarization) and would contribute if $\varepsilon$ is perpendicular to the surface ( $s$ polarization). Three distinct kinds of adsorption sites are currently identified on this surface, with the adsorbate bonded to one, two or three surface atoms.

For the various types of sites taking into account expression (3), one obtains for adsorption on top sites

$$
N_{j}=\cos ^{2} \theta
$$

for bridge sites,

$$
N_{j}=2\left(\cos ^{2} \theta \cos ^{2} \beta+\sin ^{2} \theta \sin ^{2} \beta \sin ^{2} \phi\right)
$$

and for threefold sites, on a (111) surface

$$
N_{j}=3 \cos ^{2} \theta \cos ^{2} \beta+1.5 \sin ^{2} \theta \sin ^{2} \beta \text {. }
$$

This method was applied by Shirai et al. [12] in the study of the adsorption of $\mathrm{Co}$ oxides on a (0001) $\alpha-\mathrm{Al}_{2} \mathrm{O}_{3}$ surface. The sample was prepared by vapour deposition of $\mathrm{Co}_{2}(\mathrm{CO})_{8}$ on a (0001) surface of alumina followed by an evacuation at $300 \mathrm{~K}$ and an oxidation under oxygen at $300 \mathrm{~K}$. As shown in Fig. 8, the

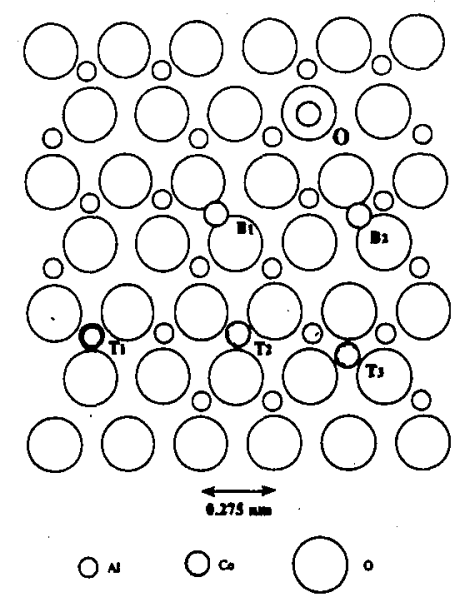

Fig. 8. The possible on top, bridge and threefold hollow sites of the alumina surface.

arrangement of the oxygen atoms on the alumina surface is hexagonal. The $\mathrm{Al}$ atoms are located in the second layer surrounded by 40 atoms. Hence, two kinds of bridge sites are possille for the location of Co atoms, B1 and B2, three types of threefold hollow sites T1, T2, T3 and only one type of top site. The results of the two EXAFS experiments in the $p$ and $s$ polarization modes are reported in Table VI. Only the model with Co atoms in the threefold hollow sites agree with the experimental data. Moreover no $\mathrm{Al}$ atoms are detected in the first coordination sphere of Co atoms, excluding the T1 and T3 sites.

Moreover, an XPS measurement at the O $1 s$ and Co $2 p$ levels lead to a Co concentration $1 / 3$ of that of the oxygen, corroborating the proposed in Fig. 9. 


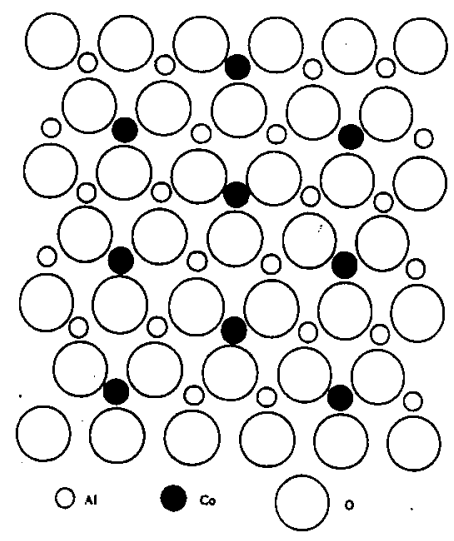

Fig. 9. Distribution of Co atoms on threefold hollow sites of the alumina (0001) surface.

\section{TABLE VI}

Comparison of EXAFS experimental data at the Co $K$-edge for the sample treated at $300 \mathrm{~K}$ and measured for two polarization modes, with several models of adsorption sites.

\begin{tabular}{c|c|c|c|c|c}
\hline \hline \multicolumn{2}{c|}{} & \multicolumn{4}{|c}{ Effective coordination number $N$} \\
\hline Polarization & Bond distance & Experimental & On top & Bridge & Threefold \\
\hline$s$ & 2.08 & 3.3 & 0 & 1.3 & 2.6 \\
$p$ & 2.08 & 3.9 & 3 & 3.3 & 3.7 \\
$p / s$ ratio & & 1.2 & $\infty$ & 2.6 & 1.4
\end{tabular}

Thus the Co atoms are engaged in monomers and bonded to three $\mathrm{O}$ atoms. Such a surface with no active oxygen atoms, was found to be little reactive in the $\mathrm{CO}$ oxidation.

In a further experiment, these authors have performed an oxidation of this sample at $800 \mathrm{~K}$ under oxygen.

The EXAFS spectra are totally modified and structures are now observed at large $R$ values, indicating the presence of several coordination spheres around Co. The results reported in Table VII showing Co-Co bond distances up to $5 \AA$, confirm the presence of larger $\mathrm{Co}-\mathrm{O}$ clusters. The authors conclude that these clusters have a diameter of $10 \AA$. However the large value of $N$ found for the 4-th coordination sphere, would indicate that the particles have rather a diameter of 40 to $60 \AA$.

In any case the presence of coordinatively unsaturated oxygen atoms, bridging two $\mathrm{Co}^{3+}$ would explain the increasing reactivity of this phase compared to the sample heated at $300 \mathrm{~K}$, in the oxidation of $\mathrm{CO}$. 


\section{TABLE VII}

EXAFS results for the sample oxidized at $800 \mathrm{~K}$. Comparison with crystallographic data of the $\mathrm{Co}_{3} \mathrm{O}_{4}$ spinel structure.

\begin{tabular}{c|c|c|c|c|c|c|c|c}
\hline \hline & \multicolumn{2}{|c|}{$\mathrm{Co}-\mathrm{O}$} & \multicolumn{2}{c|}{$\mathrm{Co}-\mathrm{Co}$} & \multicolumn{2}{c|}{$\mathrm{Co}-\mathrm{Co}$} & \multicolumn{2}{c}{$\mathrm{Co}-\mathrm{Co}$} \\
\hline Polarization & $N$ & $R$ & $N$ & $R$ & $N$ & $R$ & $N$ & $R$ \\
\hline$s$ & 4.2 & 1.94 & 6.9 & 2.86 & 9.3 & 3.42 & 3.1 & 4.96 \\
$p$ & 5.2 & 1.92 & 6 & 2.85 & 7.1 & 3.4 & 2.7 & 4.97 \\
$\mathrm{Co}_{3} \mathrm{O}_{4}$ & 5.4 & 1.95 & 6 & 2.85 & 9.3 & 3.38 & 6 & 4.95
\end{tabular}

\section{Conclusion}

These examples constitute the proof that EXAFS has now reached a maturity. For complex systems composed of several elements it is clear that a complete description of the structure and especially of the surface of the material necessitates the use of complementary methods. These limitations are partly due to the poor accuracy of the determination of the coordination numbers but also to the fact that EXAFS itself is not sensitive to the bond angles.

This difficulty can be partly overcome by using simultaneously XANES which has proved to be especially efficient to determine the mode of adsorption of molecules on surfaces.

\section{References}

[1] N. Toshima, M. Harada, T. Yonesawa, K. Kushihashi, K. Asakura, J. Phys. Chem. 95, 7448 (1991).

[2] M. Harada, K. Asakura, Y. Ueki, N. Toshima, J. Phys. Chem 97, 10742 (1993).

[3] R. Joyner, K. Minachev, P. Pudney, E. Shapiro, G. Tuleouva, Catal. Lett. 5, 257 (1990).

[4] J.F. Faudon, F. Senoq, G. Bergeret, B. Moraweck, G. Clugnet, C. Nicot, A. Renouprez, J. Catal. 144, 460 (1993).

[5] A. Borgna, B. Moraweck, J. Massardier, A. Renouprez, J. Catal. 128, 91 (1991).

[6] B.K. Teo, P.A. Lee, J. Am. Chem. Soc. 95, 7448 (1991).

[7] N. Toshima, K. Kushihashi, T. Yonezawa, H. Hirai, Chem. Lett., 1769 (1989).

[8] G.H. Via, K.F. Drake, G. Meitzner, F.W. Lytle, J. Sinfeld, Catal. Lett. 5, 25 (1990).

[9] R. Kelley, Surf. Interface Anal. 7, 1 (1987).

[10] P. Wynblatt, R.C. Ku, Surf. Sci. 65, 511 (1977).

[11] P.H. Citrin, J.E. Rowe, P. Eisenberger, Phys. Rev, B 28, 2299 (1983).

[12] M. Shirai, T. Inoue, H. Onishi, K. Asakura, Y. Iwasawa, J. Catal. 145, 159 (1994). 